\title{
Axion dark matter search using arm cavity transmitted beams of gravitational wave detectors
}

\author{
Koji Nagano $\odot,{ }^{1}$ Hiromasa Nakatsuka $\odot,{ }^{2}$ Soichiro Morisaki $\odot,{ }^{3}$ Tomohiro Fujita $\odot,{ }^{4,5}$ Yuta Michimura $\odot,{ }^{6,7}$ and Ippei Obata $\odot^{8}$ \\ ${ }^{1}$ Institute of Space and Astronautical Science, Japan Aerospace Exploration Agency, \\ Sagamihara City 252-5210, Japan \\ ${ }^{2}$ Institute for Cosmic Ray Research, University of Tokyo, Kashiwa 277-8582, Japan \\ ${ }^{3}$ Department of Physics, University of Wisconsin-Milwaukee, Milwaukee, Wisconsin 53201, USA \\ ${ }^{4}$ Waseda Institute for Advanced Study, Waseda University, Shinjuku, Tokyo 169-8050, Japan \\ ${ }^{5}$ Research Center for the Early Universe, The University of Tokyo, Bunkyo, Tokyo 113-0033, Japan \\ ${ }^{6}$ Department of Physics, University of Tokyo, Bunkyo, Tokyo 113-0033, Japan \\ ${ }^{7}$ PRESTO, Japan Science and Technology Agency (JST), Kawaguchi, Saitama 332-0012, Japan \\ ${ }^{8}$ Max-Planck-Institut für Astrophysik, Karl-Schwarzschild-Straße 1, 85741 Garching, Germany
}

(Received 4 July 2021; accepted 12 August 2021; published 16 September 2021)

\begin{abstract}
Axion is a promising candidate for ultralight dark matter which may cause a polarization rotation of laser light. Recently, a new idea of probing the axion dark matter by optical linear cavities used in the arms of gravitational wave detectors has been proposed [Phys. Rev. Lett. 123, 111301 (2019). In this article, a realistic scheme of the axion dark matter search with the arm cavity transmission ports is revisited. Since photons detected by the transmission ports travel in the cavity for odd-number of times, the effect of axion dark matter on their phases is not canceled out and the sensitivity at low-mass range is significantly improved compared to the search using reflection ports. We also take into account the stochastic nature of the axion field and the availability of the two detection ports in the gravitational wave detectors. The sensitivity to the axion-photon coupling, $g_{a \gamma}$, of the ground-based gravitational wave detector, such as Advanced LIGO, with 1-year observation is estimated to be $g_{a \gamma} \sim 3 \times 10^{-12} \mathrm{GeV}^{-1}$ below the axion mass of $10^{-15} \mathrm{eV}$, which improves upon the limit achieved by the CERN Axion Solar Telescope.
\end{abstract}

DOI: $10.1103 /$ PhysRevD.104.062008

\section{INTRODUCTION}

Axion is a hypothetical pseudo-scalar field that was proposed by Peccei and Quinn to solve the strong $C P$ problem in quantum chromodynamics (QCD) [1]. Their original idea is called "QCD axion." Besides the QCD axion, string theory also predicts a plenty of axionlike particles via the compactifications of extra dimensions [2]. Typically, QCD axion and axionlike particles have two noteworthy features. First, their masses can be much lighter than $1 \mathrm{eV}$ due to the shift symmetry. Second, they have an oscillatory feature leading to their behavior like a nonrelativistic fluid in the Universe [3]. Owing to these two features, QCD axion or axionlike particles are considered to be a leading candidate for dark matter, among the ultralight dark matter models $[4,5]$. Hereafter in this article, we jointly call them "axion."

Published by the American Physical Society under the terms of the Creative Commons Attribution 4.0 International license. Further distribution of this work must maintain attribution to the author(s) and the published article's title, journal citation, and DOI. Funded by SCOAP.
To probe the axion, a variety of experiments and observations have been performed [6-27] (see also [28] for recent review). They utilize a weak topological coupling between the axion and gauge bosons, such as photons, and detect an axion-photon conversion effect (dubbed "Primakoff effect") under an external magnetic field [29]. For example, CERN Axion Solar Telescope (CAST) probes the axions produced in the Sun by converting the axion flux into x-rays with dipole magnets [7]. Recently, some projects have also achieved almost the same limit as the CAST limit in a certain axion mass range below $\sim 1 \mathrm{neV}[13,27]$. Another approach is the use of the astronomical telescopes to detect the electromagnetic signals that is generated from the axion produced in the astronomical object, such as SN1987A. The axions produced in SN1987A can be converted to the gamma ray according to galactic magnetic field and the associated spectral modulation is potentially observable with the gamma-ray telescope [16]. Moreover, the recent observations of gravitational waves have provided the constraints independent from the above electromagnetic astrophysical observations through the measurement of spinning black hole [30].

Recently, a new search method for axion dark matter without using the Primakoff effect has been developed. The 
axion field coupled to photons behaves as a birefringent material in our universe by differentiating the phase velocities between left- and right-handed photons [31,32]. Thanks to the recent development of the optics technology, many new approaches measuring the photon's birefringence caused by axion dark matter have been proposed [33-38]. These studies have led to an idea of using the existing or planned instruments originally for the different purposes, such as laser interferometric gravitational wave detectors [39-44]. For example, there is a proposal to use long FabryPerot cavities in the gravitational-wave detectors for axion dark matter search [37]. We call this scheme ADAM-GD (axion dark matter search with gravitational wave detectors). ADAM-GD enables us to probe the axion dark matter with the mass less than $10^{-10} \mathrm{eV}$ that is a complement method to the axion dark matter search with the gravitational wave observation [30,45].

In the ADAM-GD scheme, the axion dark matter can be searched for in the reflection and the transmission port of the Fabry-Perot cavity. Of these two ports, the transmission port is more feasible than the reflection port in terms of the experimental setup since the reflection port is occupied by more main optics used for the gravitational wave detection, for example a signal extraction mirror [46].

To demonstrate the benefits from the use of transmission port, in this article we develop a realistic performance of ADAM-GD with the transmission port and reevaluate its potential sensitivity to the axion-photon coupling. In particular, we revisit the response of the phase velocity modulation in the transmission port and consider the influence of the odd-number ways of optical path in the cavity on the axion signal, which was overlooked in the previous study [37]. To make the estimate more realistic, we take the randomness of the axion dark matter particles into account and introduce the associated stochastic behavior of axion field to the signal. Moreover, we also perform the coherent analysis of two transmission ports of gravitational-wave detectors to improve the sensitivity level. Note that, in this article, we set the natural unit $\hbar=c=1$.

This article is organized as follows. Section II describes a brief summary of the phase velocity variation caused by axion dark matter and the dynamics of axion field including its stochastic behavior. In Sec. III, the Fabry-Perot cavity response to the phase velocity modulation including the odd-number optical path effect is studied. Next, Sec. IV presents a study on the coherent analysis of two detection ports. Finally, Sec. V gives expected sensitivities of the gravitational wave detectors to the axion-photon coupling and discussion for the actual search.

\section{PHASE VELOCITY MODULATION}

In this section, we revisit the dynamics of axion field background and compute its effective oscillation amplitude by including the stochastic effect which will be used to obtain the sensitivity of interferometer experiments.
The axion can couple to the photon through the ChernSimons interaction,

$$
\mathcal{L}_{I}=\frac{g_{\mathrm{a} \gamma}}{4} a(t) F_{\mu \nu} \tilde{F}^{\mu \nu},
$$

where $a(t)$ is the axion field, $g_{\mathrm{ay}}$ is the axion-photon coupling constant, $F_{\mu \nu}$ is field strength of electromagnetic field and $\tilde{F}^{\mu \nu} \equiv \epsilon^{\mu \nu \rho \sigma} F_{\rho \sigma} / 2$ is its Hodge dual with the LeviCivita antisymmetric tensor $\epsilon^{\mu \nu \rho \sigma}$. It is known that the background axion field modifies the dispersion relation of photon. Under the homogeneous axion field background, the left- (right-) circular polarized photon has a modified dispersion relation,

$$
\omega_{\mathrm{L} / \mathrm{R}}^{2}=k^{2}\left(1 \mp g_{\mathrm{a} \gamma} \dot{a} / k\right),
$$

with its momentum $k$ and angular frequency $\omega_{\mathrm{L} / \mathrm{R}}$. Phase velocities of left-handed and right-handed photons are represented as

$$
\begin{gathered}
c_{\mathrm{L} / \mathrm{R}} \equiv \frac{\omega_{\mathrm{L} / \mathrm{R}}}{k} \simeq 1 \mp \delta c(t), \\
\delta c(t) \equiv \frac{g_{\mathrm{a} \gamma} \dot{a}}{2 k} .
\end{gathered}
$$

The difference of phase velocities between circular polarization modes rotates the direction of linear polarization of photon. Therefore, the oscillating axion field converts a part of p-polarized light into s-polarized light or vice versa, which enables us to probe axion dark matter through gravitational-wave detectors, as discussed in the next section.

In the literature, the axion field is often modeled by a periodic oscillation, $a(t)=a_{0} \cos \left(m t+\theta_{0}\right)$, with a constant amplitude $a_{0}$ as well as constant phase $\theta_{0}$, where $m$ is the mass of axion. In reality, however, the axion field should be understood as a superposition of many classical waves with different velocities and phases [47]

$$
a(t)=\sum_{i} \Lambda \cos \left(m\left(1+v_{i}^{2} / 2\right) t+\theta_{i}\right),
$$

where $\Lambda, v_{i}$ and $\theta_{i}$ are amplitude, velocity and phase of $i$-th wave, respectively. The velocity dispersion of dark matter around the sun in our Galaxy is $v \sim 10^{-3}$. Note that $\Lambda$ is properly normalized such that the expectation value of the energy density, $\rho_{\mathrm{DM}}=\left(\dot{a}^{2}+m^{2} a^{2}\right) / 2$, reproduces the observed value in the local universe, $\rho_{\text {DM }} \sim 0.4 \mathrm{GeV} / \mathrm{cm}^{3}$ [48]. The oscillation frequencies of these waves are slightly different due to the velocity distribution, and the superposed axion $a(t)$ does not show a perfect coherent oscillation. Instead, the amplitude and phase of $a(t)$ varies in a so-called coherent timescale, $\tau \equiv 2 \pi /\left(m v^{2}\right)$. Therefore, after performing the summation of Eq. (5), the axion field is expressed as 


$$
a(t)=a_{0}(t) \cos \left(m t+\theta_{0}(t)\right),
$$

where $a_{0}(t)$ and $\theta_{0}(t)$ slowly change over $\tau$, while they can be approximated by constants for a shorter timescale than $\tau$. Because of this time-evolving nature, $a_{0}(t)$ may significantly deviate from its mean value

$$
\bar{a} \equiv \frac{\sqrt{2 \rho_{\mathrm{DM}}}}{m}
$$

when we conduct an experiment. Therefore, the effective axion amplitude during experiments should be carefully considered to determine the sensitivities.

To take into account the time evolving nature of $a_{0}$ and $\theta_{0}$, we approximate them by constants which stochastically change their values every coherent time $\tau$. We naively assume that the phase $\theta_{0}$ takes a value between 0 and $2 \pi$ in the equal probability. The amplitude $a_{0}$ follows the Rayleigh distribution, because the sum of the amplitudes of the waves with random phases in Eq. (5) can be seen as the distance from the origin in a random walk process in the complex plane $[47,49]$. The probability distribution of $a_{0}$ is given by

$$
P^{\text {(Ray) }}\left(a_{0}\right) \mathrm{d} a_{0} \equiv \frac{2 a_{0}}{\bar{a}^{2}} \exp \left(-\frac{a_{0}^{2}}{\bar{a}^{2}}\right) \mathrm{d} a_{0} .
$$

If the measurement time is shorter than the coherent time, $T_{\text {obs }} \lesssim \tau$, only one realization of $a_{0}$ matters and Eq. (8) suffices for our purpose.

For $T_{\text {obs }} \gtrsim \tau$, the time variations of $a_{0}$ and $\theta_{0}$ become relevant. We divide the measurement time into $N \equiv T_{\text {obs }} / \tau$ domains in which $a_{0}$ and $\theta_{0}$ can be approximated by constants with different values. We call their values in the $i$ th domain $a_{i}$ and $\theta_{i}$. Then, the axion field is approximately written as

$$
a((i-1) \tau<t<i \tau)=a_{i} \cos \left(m t+\theta_{i}\right),
$$

where $a_{i}$ and $\theta_{i}$ are constant, and $i$ runs from 1 to $N$. Using Eq. (4), we find the phase modulation in the $i$ th domain as

$$
\delta c((i-1) \tau<t<i \tau)=\frac{g_{a \gamma} m a_{i}}{2 k} \sin \left(m t+\theta_{i}\right)
$$

In the data analysis, we work in the Fourier space and sum up not the amplitude but the power of the signal over the all domains. This is because the random phase $\theta_{i}$ prevents us from coherently adding up the amplitude. In each domain, the signal power is proportional to $g_{a \gamma}^{2} a_{i}^{2}$ and its sum yields $g_{a \gamma}^{2} \sum_{i} a_{i}^{2}$. Thus, the sensitivity of our experiment is characterized by the root mean square (RMS) of $a_{i}$, namely $A_{N} \equiv \sqrt{\sum_{i} a_{i}^{2} / N}$. From Eq. (8), we find the probability distribution of $A_{N}$ as (See Appendix for derivation.)

$$
P^{(N)}\left(A_{N}\right)=\frac{2 N^{N}}{\bar{a} \Gamma(N)}\left(\frac{A_{N}}{\bar{a}}\right)^{2 N-1} e^{-N\left(A_{N} / \bar{a}\right)^{2}},
$$

where $\Gamma(n)$ is the gamma function. Note that $P^{(1)}(a)=$ $P^{\text {(Ray) }}(a)$. In the limit $N \rightarrow \infty, P^{(N)}(a) \rightarrow \delta(a-\bar{a})$ and the stochastic effect vanishes.

Given that we basically measure $g_{a \gamma}^{2} A_{N}^{2}$, the sensitivity to $g_{\mathrm{ay}}$ would be weaker if $A_{N}$ happens to be smaller during our experiment. To conservatively estimate the sensitivity by taking into account this stochastic effect, we introduce a probabilistic RMS amplitude $A_{N}^{\text {low }}(p)$ where $A_{N} \geq A_{N}^{\text {low }}(p)$ occurs with probability of $p$. For instance, we expect that the actual RMS amplitude $A_{N}$ is larger than or equal to $A_{N}^{\text {low }}(0.95)$ with a probability of $95 \%$. We compute $A_{N}^{\text {low }}(p)$ as

$p=\int_{A_{N}^{\text {low }}(p)}^{\infty} \mathrm{d} a P^{(N)}(a)=\frac{\Gamma\left(N, N\left(A_{N}^{\text {low }}(p) / \bar{a}\right)^{2}\right)}{\Gamma(N)}$,

where $\Gamma(a, z) \equiv \int_{z}^{\infty} t^{a-1} e^{-t} \mathrm{~d} t$ is the incomplete gamma function. In Fig. 1, we show $A_{N}^{\text {low }}(p)$ for $p=0.68$ and 0.95 . For $T_{\mathrm{obs}}<\tau$, we measure only $N=1$ realization of the axion amplitude, and we find $A_{1}^{\text {low }}(68 \%) \approx 0.62 \bar{a}$ and $A_{1}^{\text {low }}(95 \%) \approx 0.23 \bar{a}$. As the measurement time becomes longer, the stochastic effect becomes less important, since we average over $N$ realized values and $A_{N}^{\text {low }}(p)$ converges into $\bar{a}$ in the limit $N \rightarrow \infty$. We find fitting functions for $A_{N}^{\text {low }}(68 \%)$ and $A_{N}^{\text {low }}(95 \%)$ with respect to $N$ whose relative error is smaller than $1 \%$,

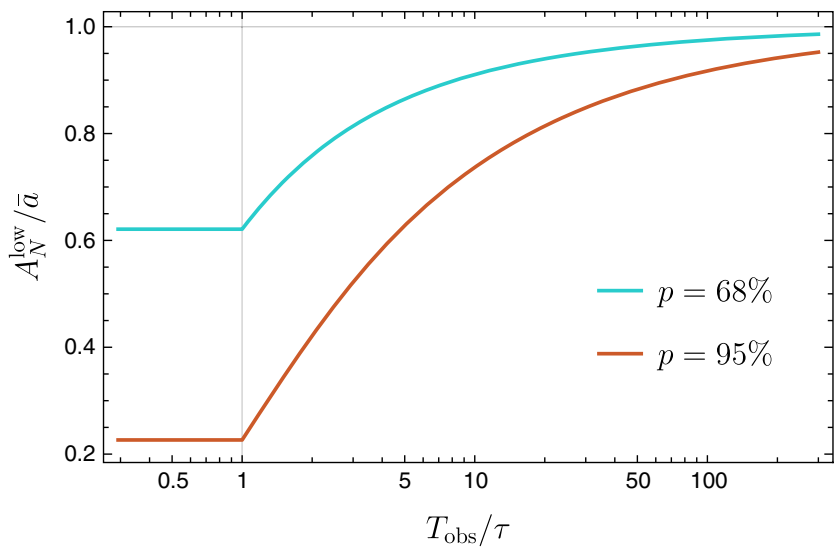

FIG. 1. The probabilistic amplitude of axion field $A_{N}^{\text {low }}(p)$ given in Eq. (12). We expect the actual axion RMS amplitude $A_{N}$ is larger than or equal to $A_{N}^{\text {low }}(p)$ by probabilities of $p=68 \%$ (Cyan) and 95\% (Red). The horizontal axis denotes the experiment time divided by the coherent time, $T_{\text {obs }} / \tau$, which corresponds to the number of time domain $N$ for $T_{\text {obs }} / \tau>1$, while $N$ remains unity for $T_{\mathrm{obs}} / \tau<1$. 


$$
\begin{aligned}
& \frac{A_{N}^{\text {low }}(68 \%)}{\bar{a}} \simeq\left\{\begin{array}{ll}
0.621 & (N<1) \\
1-0.145 N^{-0.956}-0.234 N^{-1 / 2} & (1<N)
\end{array},\right. \\
& \frac{A_{N}^{\text {low }}(95 \%)}{\bar{a}} \simeq \begin{cases}0.226 & (N<1) \\
1+0.049 N^{-3.410}-0.822 N^{-1 / 2} & (1<N)\end{cases}
\end{aligned}
$$

Wrapping up the above arguments, we describe the way to find the sensitivity. In the experiment, we measure the oscillation amplitude $\delta c_{0}=g_{a \gamma} m a_{0}(t) / 2 k$ of the phase velocity difference in Eq. (4). We then process this data into $g_{a \gamma} m A_{N} / 2 k$ by dividing it into $N=T_{\text {obs }} / \tau$ domains. However, we cannot determine the axion RMS amplitude $A_{N}$ due to the stochastic effect. Then we replace $A_{N}$ by $A_{N}^{\text {low }}(p)$ and estimate the sensitivity based on

$$
g_{a \gamma}=\frac{2 k \delta c_{0}}{m A_{N}^{\operatorname{low}}(p)} .
$$

The actual sensitivity to $g_{a \gamma}$ is better than or equal to the value estimated by the above equation by a probability of $p$.

Note that once we have performed the experiment and found null detection, we could put the upper bound of coupling constant using a more dedicated treatment [49].

\section{AXION SEARCH WITH AN ARM CAVITY TRANSMITTED BEAMS OF GRAVITATIONAL WAVE DETECTORS}

In this section, we express the sensitivity of the transmission port of the gravitational wave detector. First, we consider the response function for the transmission port. The setup is shown in Fig. 2(a). The laser light with a wavelength $\lambda=2 \pi / k$ is injected to the optical cavity in purely p-polarized light. As explained in the previous section, the axion field generates s-polarized signal light from the p-polarized light. The optical cavity is composed of the input and the end mirror. The input (end) mirror has reflectivity and transmissivity of $\left(r_{1}, t_{1}\right) \quad\left(\left(r_{2}, t_{2}\right)\right)$. Hereafter, we assume the optical cavity meets the resonant condition. In other words, the cavity length, $L_{\text {cav }}$, is kept to be integer multiple of the half wavelength of the laser light with, for example, Pound-Drever-Hall technique [50]. In this condition, the laser light including the s-polarized light of the axion signal is enhanced in the cavity as explained later. The enhanced signal is observed in the detection port at the cavity transmission. The detection port consists of the light polarization detector with a half-wave plate (HWP) and a polarizing beam splitter (PBS).

Here, we explain how the laser light is enhanced in the cavity. The input laser light is expressed as

$$
\boldsymbol{E}_{\text {in }}(t)=\boldsymbol{E}^{\mathrm{p}}(t)=E_{0} e^{i k t}\left(\boldsymbol{e}^{L} \boldsymbol{e}^{R}\right) \frac{1}{\sqrt{2}}\left(\begin{array}{l}
1 \\
1
\end{array}\right),
$$

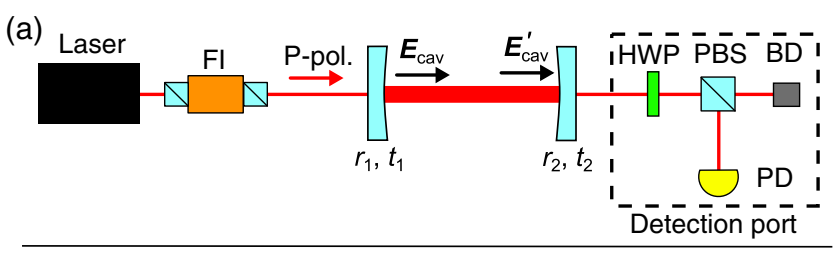

(b)

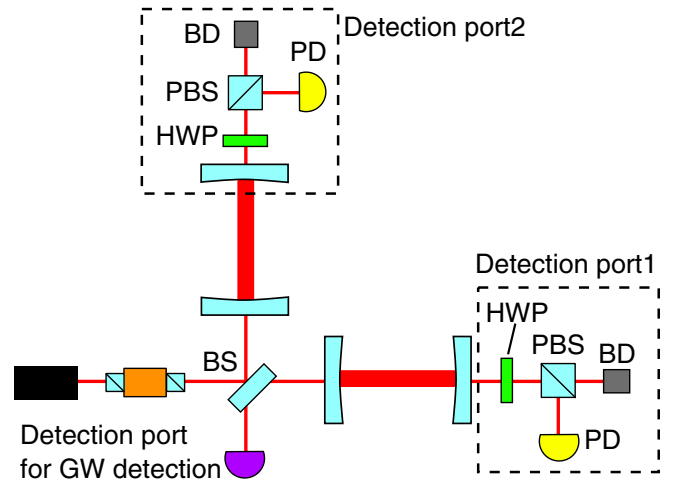

FIG. 2. Schematic of experimental setup for axion search with (a) a linear optical cavity and (b) a gravitational wave detector. FI, Faraday isolator; HWP, half wave plate; PBS, polarizing beam splitter; PD, photodetector; BD, beam dump; BS, beam splitter. In the detection port at the cavity transmission, polarization analysis is performed with the HWP, PBS, and PD, and the axion signal can be detected. Components for phase measurement are not shown. Two PBSs in FI are placed rotated by 45 degrees along the optical path.

where $\boldsymbol{E}^{\mathrm{p}}(t)$ is the electric vector of the p-polarized light, and $\boldsymbol{e}^{L}$ and $\boldsymbol{e}^{R}$ are basis vectors of the left- and right-handed photon, respectively. When the cavity is in the resonant condition, the electric field of the input laser light is enhanced by $\frac{1}{1-r_{1} r_{2}}$ [51]. In the presence of the background axion field, the left- and right-handed light has the phase variation with opposite sign. Therefore, the intracavity electric field at the input mirror, $\boldsymbol{E}_{\text {cav }}(t)$, is denoted as [37],

$$
\begin{aligned}
\boldsymbol{E}_{\mathrm{cav}}(t)= & \frac{t_{1} E_{0} e^{i k t}}{1-r_{1} r_{2}}\left(\begin{array}{ll}
\boldsymbol{e}^{\mathrm{L}} & \boldsymbol{e}^{\mathrm{R}}
\end{array}\right) \\
& \times\left(\begin{array}{cc}
1+i \delta \phi(t) & 0 \\
0 & 1-i \delta \phi(t)
\end{array}\right) \frac{1}{\sqrt{2}}\left(\begin{array}{l}
1 \\
1
\end{array}\right) \\
& =\frac{t_{1}}{1-r_{1} r_{2}}\left[\boldsymbol{E}^{\mathrm{p}}(t)-\delta \phi(t) \boldsymbol{E}^{\mathrm{s}}(t)\right],
\end{aligned}
$$

where $\boldsymbol{E}^{\mathrm{s}}(t)$ is the electric vector of the s-polarized light represented as

$$
\boldsymbol{E}^{\mathrm{s}}(t)=E_{0} e^{i k t}\left(\boldsymbol{e}^{L} \boldsymbol{e}^{R}\right) \frac{1}{\sqrt{2} i}\left(\begin{array}{c}
1 \\
-1
\end{array}\right)
$$

and $\delta \phi(t)$ is the polarization angle rotated by the phase velocity deference $\delta c$, 


$$
\delta \phi(t) \equiv \int_{-\infty}^{\infty} \tilde{\delta c}(m) H_{\mathrm{a}}(m) e^{i m t} \frac{d m}{2 \pi} .
$$

Here, $\tilde{\delta c}(m)$ is the Fourier transformation of $\delta c(t), \delta c(t) \equiv$ $\int_{-\infty}^{\infty} \tilde{\delta c}(m) e^{i m t} \frac{d m}{2 \pi}$ and $H_{\mathrm{a}}(m)$ is a response function of cavity,

$$
H_{\mathrm{a}}(m) \equiv i \frac{k}{m} \frac{4 r_{1} r_{2} \sin ^{2}\left(\frac{m L_{\mathrm{cav}}}{2}\right)}{1-r_{1} r_{2} e^{-i 2 m L_{\mathrm{cav}}}}\left(-e^{-i m L_{\mathrm{cav}}}\right),
$$

which corresponds to the response at the reflection port (the detection port nearby the front mirror) [37].

The intracavity electric field at the end mirror, $\boldsymbol{E}_{\mathrm{cav}}^{\prime}(t)$, is obtained by applying the transfer matrix for one way translation, $T(t)$, to that at the input mirror. $\boldsymbol{E}_{\mathrm{cav}}^{\prime}(t)$ is represented as

$$
\begin{aligned}
\boldsymbol{E}_{\mathrm{cav}}^{\prime}\left(t+L_{\mathrm{cav}}\right)= & \frac{t_{1} E_{0} e^{i k t}}{1-r_{1} r_{2}}\left(\begin{array}{ll}
\boldsymbol{e}^{\mathrm{L}} & \boldsymbol{e}^{\mathrm{R}}
\end{array}\right) T\left(t+L_{\mathrm{cav}}\right) \\
& \times\left(\begin{array}{cc}
1+i \delta \phi(t) & 0 \\
0 & 1-i \delta \phi(t)
\end{array}\right) \frac{1}{\sqrt{2}}\left(\begin{array}{l}
1 \\
1
\end{array}\right)
\end{aligned}
$$

where

$$
T(t) \equiv\left(\begin{array}{cc}
e^{-i \phi^{\mathrm{L}}(t)} & 0 \\
0 & e^{-i \phi^{\mathrm{R}}(t)}
\end{array}\right)
$$

$$
\phi^{\mathrm{L} / \mathrm{R}}(t) \equiv k L_{\mathrm{cav}} \mp k \int_{t-L_{\mathrm{cav}}}^{t} \delta c\left(t^{\prime}\right) d t^{\prime}
$$

Notice that, since it takes $L_{\mathrm{cav}}$ for the electric field to travel from the input mirror to the end mirror, we have to consider $\boldsymbol{E}_{\text {cav }}^{\prime}\left(t+L_{\text {cav }}\right)$ if we start from $\boldsymbol{E}_{\text {cav }}(t)$. When we assume $\left|\delta \phi^{\prime}(t)\right| \ll 1$ and $2 k L_{\text {cav }}=2 \pi l(l \in \mathbb{N})$ (resonant condition of the cavity), Eq. (22) can be deformed as

$$
T(t) \simeq(-1)^{l}\left(\begin{array}{cc}
1+i \delta \phi^{\prime}(t) & 0 \\
0 & 1-i \delta \phi^{\prime}(t)
\end{array}\right)
$$

Consequently, Eq. (21) is denoted as

$$
\begin{aligned}
\boldsymbol{E}_{\mathrm{cav}}^{\prime}(t+L) & \simeq(-1)^{l} \frac{t_{1} E_{0} e^{i k t}}{1-r_{1} r_{2}}\left(\boldsymbol{e}^{\mathrm{L}} \boldsymbol{e}^{\mathrm{R}}\right)\left(\begin{array}{cc}
1+i\left(\delta \phi(t)+\delta \phi^{\prime}\left(t+L_{\mathrm{cav}}\right)\right) & 0 \\
0 & 1-i\left(\delta \phi(t)+\delta \phi^{\prime}\left(t+L_{\mathrm{cav}}\right)\right)
\end{array}\right) \frac{1}{\sqrt{2}}\left(\begin{array}{l}
1 \\
1
\end{array}\right), \\
& =(-1)^{l} \frac{t_{1}}{1-r_{1} r_{2}}\left[\boldsymbol{E}^{\mathrm{p}}(t)-\left(\delta \phi(t)+\delta \phi^{\prime}\left(t+L_{\mathrm{cav}}\right)\right) \boldsymbol{E}^{\mathrm{s}}(t)\right] .
\end{aligned}
$$

Here, the second or higher order terms of $\delta \phi(t)$ and $\delta \phi^{\prime}(t)$ are ignored.

From Eq. (23), $\delta \phi^{\prime}(t)$ is expressed as

$$
\begin{gathered}
\delta \phi^{\prime}\left(t+L_{\mathrm{cav}}\right)=k \int_{t}^{t+L_{\mathrm{cav}}} \delta c\left(t^{\prime}\right) d t^{\prime} \\
=k \int_{t}^{t+L_{\mathrm{cav}}} \int_{-\infty}^{\infty} \tilde{\delta c}(m) e^{i m t^{\prime}} \frac{d m}{2 \pi} d t^{\prime} \\
\equiv \int_{-\infty}^{\infty} \tilde{\delta c}(m) H_{\mathrm{a}}^{\prime}(m) e^{i m t} \frac{d m}{2 \pi} \\
H_{\mathrm{a}}^{\prime}(m)=\frac{2 k}{m} e^{i \frac{m L_{\mathrm{cav}}}{2}} \sin \left(\frac{m L_{\mathrm{cav}}}{2}\right) .
\end{gathered}
$$

As a result, a total response function for the transmission port is defined as

$$
H_{\mathrm{a}}^{\operatorname{Trans}}(m) \equiv H_{\mathrm{a}}(m)+H_{\mathrm{a}}^{\prime}(m) .
$$

Figure 3 shows the absolute value of the response function of the transmission and reflection port. Here, we use the parameter set of DECIGO shown in Table I. As has been found in the previous work [37], the response at the reflection port $H_{\mathrm{a}}(\mathrm{m})$ degrades the sensitivity for the low mass range because the phase modulation is canceled out between the going and returning ways of optical path in the cavity. This situation is caused by the parity transformation at the reflection of mirrors. On the other hand, remarkably, the transmission port exhibits a sizable response for the low mass range. This is because the cancellation of the phase velocity modulation does not occur due to the odd-number ways of optical path in the cavity. Therefore, the use of transmission port is highly advantageous in comparison with that of reflection port for the low-mass axion searches. 


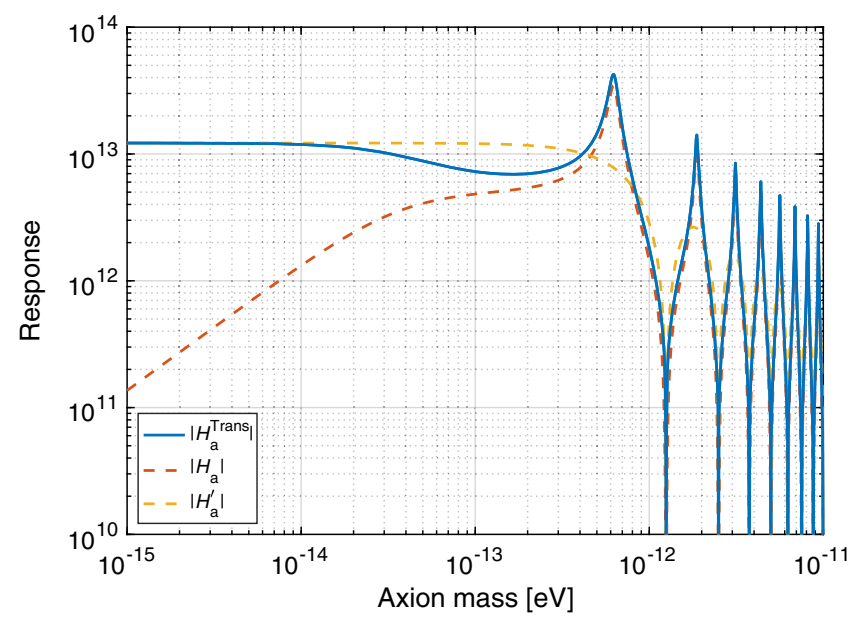

FIG. 3. Response functions for the transmission port $H^{\text {Trans }}$ (blue) of DECIGO. $H^{\text {Trans }}$ has two contributions, $H_{a}(m)$ (red dashed) and $H_{a}^{\prime}$ (yellow dashed) given in Eqs. (20) and (30), respectively.

TABLE I. Parameters of considered gravitational wave detectors. Note that $P_{0}$ is the input beam power to the cavity enhanced by the power recycling cavity for the KAGRA-like, the aLIGOlike, and the CE-like detector [52].

\begin{tabular}{lcccc}
\hline \hline Similar detector & $\begin{array}{c}L_{\text {cav }} \\
{[\mathrm{m}]}\end{array}$ & $\begin{array}{c}P_{0} \\
{[\mathrm{~W}]}\end{array}$ & $\begin{array}{c}\lambda \\
{\left[\times 10^{-9} \mathrm{~m}\right]}\end{array}$ & $\left(t_{1}^{2}, t_{2}^{2}\right)[\mathrm{ppm}]$ \\
\hline KAGRA [41] & $3 \times 10^{3}$ & 335 & 1064 & $\left(4 \times 10^{3}, 7\right)$ \\
aLIGO [39] & $4 \times 10^{3}$ & 2600 & 1064 & $\left(1.4 \times 10^{4}, 5\right)$ \\
CE [43] & $4 \times 10^{4}$ & 600 & 1550 & $\left(1.2 \times 10^{3}, 5\right)$ \\
DECIGO [44] & $10^{6}$ & 5 & 515 & $\left(3.1 \times 10^{5}\right.$, \\
& & & & $\left.3.1 \times 10^{5}\right)$ \\
\hline \hline
\end{tabular}

\section{COHERENT ANALYSIS OF TWO DETECTION PORTS}

For detectors and mass range we consider in this study, the coherent length of the axion field $v \tau \sim 10^{6} \mathrm{~m} \times$ $\left(10^{-9} \mathrm{eV} / \mathrm{m}\right)$ is larger than the sizes of detectors. In this case, the two detection ports of a detector observe the signal with common phase. We show that coherently analysing them improves the sensitivity. If $T_{\mathrm{obs}} \ll \tau$, the signal is concentrated in a single frequency bin. The Fourier component of data from the $i$ th port at that frequency bin is given by

$$
d_{i}=s+n_{i} \quad(i=1,2),
$$

where $s$ is the axion signal and $n_{i}$ is the noise of the $i$ th port. If we only had the first port, we would use the following single-port detection statistic,

$$
\rho_{1} \equiv\left|d_{1}\right|^{2}
$$

In the absence of signal, the probability distribution of $\rho_{1}$ is given by

$$
p\left(\rho_{1}\right)=\frac{1}{2 S} e^{-\frac{\rho_{1}}{2 S}}
$$

where we assume that the noise is stationary and Gaussian, and $S \equiv\left\langle\left(\Re n_{1}\right)^{2}\right\rangle=\left\langle\left(\Im n_{1}\right)^{2}\right\rangle$.

Since we have two ports observing the same signal, we consider the coherent sum as detection statistic,

$$
\rho \equiv\left|d_{1}+d_{2}\right|^{2} .
$$

The contributions from the signal to $\rho$ are 4 times larger than those to $\rho_{1}$. In the absence of signal, $\rho$ follows the same distribution as (34) with $S$ replaced by $2(S+C)$, where we assume the variances of $n_{1}$ and $n_{2}$ are the same, and $C \equiv\left\langle\Re n_{1} \Re n_{2}\right\rangle=\left\langle\Im n_{1} \Im n_{2}\right\rangle$. It means the noise contributions to $\rho$ are larger than those to $\rho_{1}$ by a factor of $2(1+C / S)$. Thus, the signal-to-noise ratio (SNR) becomes larger by a factor of $2 /(1+C / S)$. Since $\rho_{1}$ and $\rho$ are proportional to the square of the amplitude of signal, the constraints on the amplitude are improved by a factor of $\sqrt{2 /(1+C / S)}$. Hence, it is improved by $\sqrt{2}$ in the uncorrelated case, $C=0$.

If $T_{\mathrm{obs}} \gtrsim \tau$, the sum of detection statistic over multiple frequency bins or segments whose durations are shorter than $\tau$ needs to be calculated [53]. Even in this case, we can compute the coherent detection statistic instead of the single-port one for each frequency bin or segment, and take their sum. It leads to an improvement in the sensitivity by the same factor as that for $T_{\mathrm{obs}} \ll \tau$. In conclusion, the constraints on the amplitude, or the coupling constant, are improved by a factor of $\sqrt{2 /(1+C / S)}$ thanks to the coherent analysis.

\section{EXPERIMENTAL SENSITIVITY}

Based on the discussions in the previous sections, we find the sensitivity to the axion-photon coupling in ADAMGD scheme of transmission port. We assume that the primary source of noise is the quantum shot noise. Other technical noises are discussed later. First, the sensitivity of the one transmission port is considered as shown in Fig. 2 (a). We can estimate the shot noise for the axion dark matter search by estimating the amount of vacuum fluctuation of electric field in the photo detector [54]. Specifically, the shot noise spectrum, $S_{\text {shot }}$, equivalent to $\tilde{\delta c}(m)$ is estimated from the ratio of the s-polarized field generated by axion dark matter to the vacuum fluctuation in the cavity transmission. Using Eq. (26), $S_{\text {shot }}$ is denoted as

$$
\sqrt{S_{\text {shot }}(m)}=\frac{1}{\frac{t_{1} t_{2}}{1-r_{1} r_{2}} H_{\mathrm{a}}^{\operatorname{Trans}}(m) E_{0}}
$$




$$
=\frac{1}{\frac{t_{1} t_{2}}{1-r_{1} r_{2}} H_{\mathrm{a}}^{\text {Trans }}(m) \sqrt{\frac{2 P_{0}}{k}}},
$$

where $P_{0}$ is the power incident to the cavity. Here, we have used the fact that the spectrum of the vacuum fluctuation is unity. Note that the dimension of the electric field is $[\sqrt{\mathrm{Hz}}]$ in common with [54].

The SNR of $\delta c_{0}$ depends on whether the observation time is longer than the axion oscillation coherent time or not as [55]

$$
\mathrm{SNR}=\left\{\begin{array}{ll}
\frac{\sqrt{T_{\mathrm{obs}}}}{2 \sqrt{S_{\mathrm{shot}}(m)}} \delta c_{0} & \left(T_{\mathrm{obs}} \lesssim \tau\right) \\
\frac{\left(T_{\mathrm{obs}} \tau\right)^{1 / 4}}{2 \sqrt{S_{\mathrm{shot}}(m)}} \delta c_{0} & \left(T_{\mathrm{obs}} \gtrsim \tau\right)
\end{array} .\right.
$$

If we set detection threshold as the unity SNR, we can denote the detection limit of $\delta c_{0}$ as

$$
\delta c_{0} \simeq\left\{\begin{array}{ll}
\frac{2}{\sqrt{T_{\mathrm{obs}}}} \sqrt{S_{\text {shot }}(m)} & \left(T_{\mathrm{obs}} \lesssim \tau\right) \\
\frac{2}{\left(T_{\mathrm{obs}} \tau\right)^{1 / 4}} \sqrt{S_{\text {shot }}(m)} & \left(T_{\mathrm{obs}} \gtrsim \tau\right)
\end{array} .\right.
$$

Considering the stochastic effect shown in Eq. (14) and Fig. 1, the sensitivity to $g_{\mathrm{a} y}$ of one cavity is expressed as

$$
\begin{aligned}
g_{\mathrm{ay}}(m) \simeq & 1.9 \times 10^{12} \mathrm{GeV}^{-1}\left(\frac{1064 \mathrm{~nm}}{\lambda}\right) \\
& \times \frac{\bar{a}}{A_{N}^{\text {low }}(68 \%)}\left\{\begin{array}{ll}
\sqrt{\frac{\sqrt{S_{\text {shot }}(m)}}{T_{\mathrm{obs}}}} & \left(T_{\mathrm{obs}} \lesssim \tau\right) \\
\frac{\sqrt{S_{\text {shot }}(m)}}{\left(T_{\mathrm{obs}} \tau\right)^{1 / 4}} & \left(T_{\mathrm{obs}} \gtrsim \tau\right)
\end{array} .\right.
\end{aligned}
$$

Note that the unity SNR corresponds to $p=0.68$.

Since the shot noise of the two detection ports are uncorrelated, the sensitivity to $g_{\mathrm{a} \gamma}$ can be improved by a factor of $\sqrt{2}$ using the coherent analysis shown in Sec. IV. Thus, the sensitivity to $g_{\mathrm{a} y}$ of the gravitational wave detectors is represented as

$$
\begin{aligned}
g_{\mathrm{ay}}(m) \simeq & 1.9 \times 10^{12} \mathrm{GeV}^{-1}\left(\frac{1064 \mathrm{~nm}}{\lambda}\right) \\
& \times \frac{\bar{a}}{\sqrt{2} A_{N}^{\text {low }}(68 \%)}\left\{\begin{array}{ll}
\sqrt{\frac{S_{\text {shot }}(m)}{T_{\text {obs }}}} & \left(T_{\text {obs }} \lesssim \tau\right) \\
\frac{\sqrt{S_{\text {shot }}(m)}}{\left(T_{\text {obs }} \tau\right)^{1 / 4}} & \left(T_{\text {obs }} \gtrsim \tau\right)
\end{array} .\right.
\end{aligned}
$$

Figure 4 presents the estimated sensitivity to $g_{\mathrm{ay}}$ of the gravitational wave detectors as shown in Fig. 2(b) with the parameter sets given in Table I. We consider similar

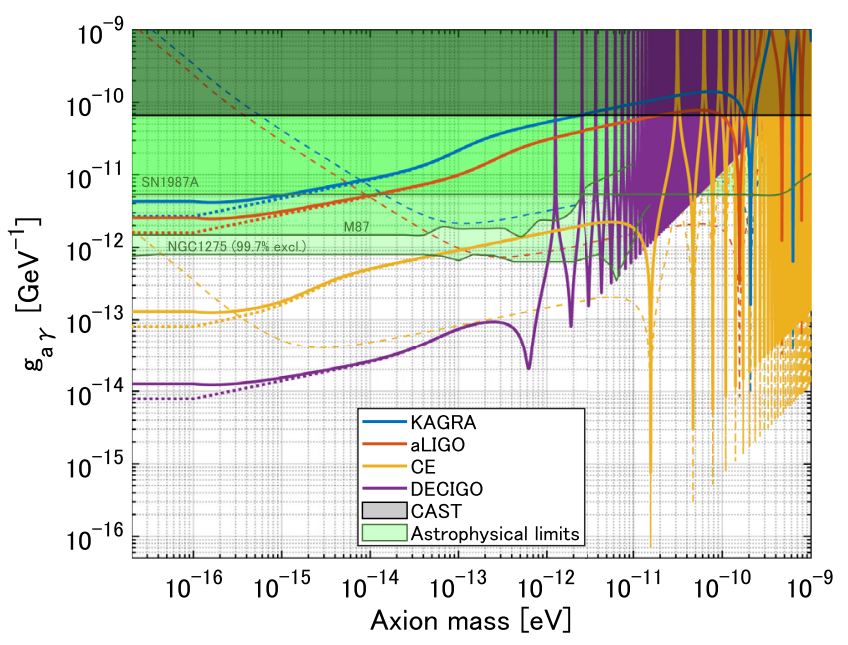

FIG. 4. Sensitivity comparison of the several parameter sets shown in table I. The dotted lines represent the sensitivity without modification of the stochastic effect. Although the higher mass range seems to be filled, they have sensitivity peaks at mass of $m=\pi(2 N-1) / L_{\text {cav }}(N \in \mathbb{N})$. The gray and green band express the current limits provided by CAST [7] and the astrophysical observations (SN1987A [16], M87 [18], and NGC 1275 [19]). The dashed lines are sensitivities using reflection port shown in [37] with modification of the stochastic effect.

parameters to the existing or planned detectors, KAGRA [41], Advanced LIGO (aLIGO) [39], Cosmic Explorer (CE) [43], and DECIGO [44]. Here, we assume 1-year observation and axion is a dominant component of dark matter. The kink around $10^{-16} \mathrm{eV}$ is due to the phase velocity modulation of the axion oscillation. With the DECIGO-like detector, the sensitivity is estimated to be $g_{\mathrm{a} \gamma} \simeq 1.3 \times 10^{-14} \mathrm{GeV}^{-1}$ around $m<10^{-16} \mathrm{eV}$. The sensitivity of the KAGRA-like (aLIGO-like) detector with transmission port overcomes the CAST limit in mass range less than $2 \times 10^{-12}\left(2 \times 10^{-11}\right) \mathrm{eV}$. The sensitivity of the CE-like detector is better than $g_{\mathrm{ay}}=2 \times 10^{-13} \mathrm{GeV}^{-1}$ in the mass range less than $10^{-15} \mathrm{eV}$. In addition, Fig. 4 shows that the observation with the transmission port in KAGRA-like, aLIGO-like, and CE-like detectors complements that with the reflection port shown in [37]. Figure 4 indicates that the sensitivity to $g_{\mathrm{a} y}$ of the ground-based detectors are almost comparable to the observation of SN1987A [16] below $\sim 10^{-15}-10^{-14} \mathrm{eV}$ and a bit weaker than the limit from the observations of M87 [18] and NGC 1275 [19] even below $10^{-16} \mathrm{eV}$. The observations with the ground-based detectors are complementary to the astronomical observations since our method is independent of astronomical model assumptions.

We note that Fig. 4 shows the shot-noise-limited sensitivities, and various practical noises should be further investigated. For example, the polarization rotation of the input laser is the possible noise source although the effect of the noise from the outside of the cavity is 
suppressed by a factor of finesse. In addition, any fluctuations of the input laser, including the fluctuations of the polarization, intensity, beam shape, and so on, could be noise sources via practical and spurious couplings. However, the Faraday isolator in the input optical path can suppress the polarization fluctuation. The fluctuations of the intensity and beam shape are (or will be) also passively filtered by the input mode cleaner, especially, in the ground-based gravitational wave detector. Nonetheless, to estimate the sensitivity of each detector, the practical noise should be taken into account by characterizing real detector performances. When the noise affects the two ports in a correlated manner, i.e., $C \neq 0$, the sensitivity may not be improved by the coherent analysis shown in Sec. IV. It is worth noting that the displacement of the cavity can not be a noise source in principle since the displacement does not make polarization rotation ideally.

\section{CONCLUSION}

In this work, we revisited ADAM-GD scheme with arm cavity transmitted beams and estimated the sensitivity to the axion-photon coupling including the stochastic effect of the axion field oscillation. We found that the use of transmission port can avoid the cancellation of photon's phase modulation caused by the parity-flipping effect of mirrors and therefore achieves the great sensitivity level for the lower mass range of axion dark matter. In addition, we analyzed the sensitivity improvement using the two detection ports that is intrinsic to the gravitational wave detectors. Then, we found that the sensitivity is improved by a factor of $\sqrt{2}$ with the coherent analysis if the noise of two detection ports are uncorrelated. In the end, the estimated sensitivity of the ground-based detector, such as aLIGO, with 1-year observation can be $g_{a \gamma} \sim 3 \times$ $10^{-12} \mathrm{GeV}^{-1}$ below $m<10^{-15} \mathrm{eV}$ and potentially overcomes the CAST limit in $m<2 \times 10^{-11} \mathrm{eV}$. We believe that this study leads to the realization of the axion dark matter search with the real gravitational wave detectors.

\section{ACKNOWLEDGMENTS}

We would like to thank Masahiro Ibe for inspiring discussions. This work was supported by the JSPS KAKENHI Grants No. JP20J01928, No. JP19J21974, No. JP18H01224, No. JP20H05850, No. JP20H05854, No. JP20H05859, No. JP17J09103, JST PRESTO Grant No. JPMJPR200B, and No. NSF PHY-1912649. K. N. acknowledges the support from JSPS Research Fellowship. H. N. acknowledges the support from the Advanced Leading Graduate Course for Photon Science. I. O. acknowledges the support from JSPS Overseas Research Fellowship.

\section{APPENDIX: PROBABILITY DISTRIBUTION OF $A_{N}$}

The derivation of $P^{(N)}\left(A_{N}\right)$ is explained in this appendix. The probability distribution of $A_{N}^{2}$ is given by

$$
\begin{aligned}
\tilde{P}^{(N)}\left(A_{N}^{2}\right) & =\int_{0}^{\infty}\left(\prod_{i} \mathrm{~d} a_{i} P^{(\text {Ray })}\left(a_{i}\right)\right) \delta\left(A_{N}^{2}-\frac{1}{N} \sum_{i} a_{i}^{2}\right) \\
& =\int_{0}^{\infty}\left(\prod_{i} \mathrm{~d} b_{i} \mathrm{e}^{-b_{i}}\right) \delta\left(A_{N}^{2}-\frac{\bar{a}^{2}}{N} \sum_{i} b_{i}\right),
\end{aligned}
$$

where $b_{i} \equiv a_{i}^{2} / \bar{a}^{2}$. After the delta function is replaced by the following integral representation,

$$
\delta(x)=\frac{1}{2 \pi} \int_{-\infty}^{\infty} \mathrm{e}^{\mathrm{i} k x} \mathrm{~d} k
$$

the integrations with respect to $b_{i}$ can be easily performed,

$$
\begin{aligned}
\tilde{P}^{(N)}\left(A_{N}^{2}\right) & =\frac{1}{2 \pi} \int_{-\infty}^{\infty} \mathrm{d} k \mathrm{e}^{\mathrm{i} k A_{N}^{2}} \prod_{i}\left(\int_{0}^{\infty} \mathrm{d} b_{i} \mathrm{e}^{-\left(1+\mathrm{i} k \bar{a}^{2} / N\right) b_{i}}\right) \\
& =\frac{1}{2 \pi \mathrm{i}^{N}}\left(\frac{N}{\bar{a}^{2}}\right)^{N} \int_{-\infty}^{\infty} \mathrm{d} k \frac{\mathrm{e}^{\mathrm{i} k A_{N}^{2}}}{\left(k-\mathrm{i} N / \bar{a}^{2}\right)^{N}} .
\end{aligned}
$$

The integrand has a $N$ th order pole at $k=\mathrm{i} N / \bar{a}^{2}$, and the integral can be evaluated with the residue theorem,

$$
\int_{-\infty}^{\infty} \mathrm{d} k \frac{\mathrm{e}^{\mathrm{i} k A_{N}^{2}}}{\left(k-\mathrm{i} N / \bar{a}^{2}\right)^{N}}=\frac{2 \pi \mathrm{i}^{N} A_{N}^{2(N-1)}}{\Gamma(N)} \mathrm{e}^{-N\left(A_{N} / \bar{a}\right)^{2}}
$$

Thus, the probability distribution of $A_{N}^{2}$ is given by

$$
\tilde{P}^{(N)}\left(A_{N}^{2}\right)=\frac{N^{N} A_{N}^{2(N-1)}}{\Gamma(N) \bar{a}^{2 N}} \mathrm{e}^{-N\left(A_{N} / \bar{a}\right)^{2}}
$$

and that of $A_{N}$ is given by

$$
\begin{aligned}
P^{(N)}\left(A_{N}\right) & =2 A_{N} \tilde{P}^{(N)}\left(A_{N}^{2}\right) \\
& =\frac{2 N^{N}}{\bar{a} \Gamma(N)}\left(\frac{A_{N}}{\bar{a}}\right)^{2 N-1} \mathrm{e}^{-N\left(A_{N} / \bar{a}\right)^{2}} .
\end{aligned}
$$

We also comment on the $N$ dependence of the $A_{N}^{\text {low }}(p)$ given by Eq. (12). In the large $N$ limit, the probability distribution of $A_{N}^{2}$ becomes Gaussian distribution, and the variance of distribution decreases as $N^{-1 / 2}$. As the probability distribution of $A_{N}$ converges into the sharp peak, the $A_{N}^{\text {low }}(p)$ also converges to the $\bar{a}$. Thus, the fitting formula in Eq. (13) includes the term proportional to $N^{-1 / 2}$ and correction term, which decreases quicker than $N^{-1 / 2}$ for large $N$. 
[1] R. D. Peccei and H. R. Quinn, Phys. Rev. Lett. 38, 1440 (1977); S. Weinberg, Phys. Rev. Lett. 40, 223 (1978); F. Wilczek, Phys. Rev. Lett. 40, 279 (1978).

[2] P. Svrcek and E. Witten, J. High Energy Phys. 06 (2006) 051; J. P. Conlon, J. High Energy Phys. 05 (2006) 078; A. Arvanitaki, S. Dimopoulos, S. Dubovsky, N. Kaloper, and J. March-Russell, Phys. Rev. D 81, 123530 (2010); L. Visinelli and S. Vagnozzi, arXiv:1809.06382.

[3] J. Preskill, M. B. Wise, and F. Wilczek, Phys. Lett. 120B, 127 (1983); L. F. Abbott and P. Sikivie, Phys. Lett. 120B, 133 (1983); M. Dine and W. Fischler, Phys. Lett. 120B, 137 (1983); P. Arias, D. Cadamuro, M. Goodsell, J. Jaeckel, J. Redondo, and A. Ringwald, J. Cosmol. Astropart. Phys. 06 (2012) 013.

[4] D. J. E. Marsh, Phys. Rep. 643, 1 (2016).

[5] E. G. M. Ferreira, arXiv:2005.03254.

[6] C. Hagmann et al. (ADMX Collaboration), Phys. Rev. Lett. 80, 2043 (1998); S. Moriyama et al., Phys. Lett. B 434, 147 (1998); T. M. Shokair et al., Int. J. Mod. Phys. A 29, 1443004 (2014); B. T. McAllister et al., Phys. Dark Universe 18, 67 (2017); D. Horns et al., J. Cosmol. Astropart. Phys. 04 (2013) 016; A. Caldwell et al. (MADMAX Working Group), Phys. Rev. Lett. 118, 091801 (2017).

[7] K. Zioutas et al. (CAST Collaboration), Phys. Rev. Lett. 94, 121301 (2005); V. Anastassopoulos et al. (CAST Collaboration), Nat. Phys. 13, 584 (2017).

[8] J. K. Vogel et al., arXiv:1302.3273.

[9] K. Ehret et al. (ALPS Collaboration), Nucl. Instrum. Methods Phys. Res., Sect. A 612, 83 (2009); R. Bähre et al., J. Instrum. 8, T09001 (2013).

[10] M. Betz, F. Caspers, M. Gasior, M. Thumm, and S. W. Rieger, Phys. Rev. D 88, 075014 (2013).

[11] H. Tam and Q. Yang, Phys. Lett. B 716, 435 (2012).

[12] P. Sikivie, N. Sullivan, and D. B. Tanner, Phys. Rev. Lett. 112, 131301 (2014).

[13] Y. Kahn, B. R. Safdi, and J. Thaler, Phys. Rev. Lett. 117, 141801 (2016); C. P. Salemi, J. W. Foster, J. L. Ouellet, A. Gavin, K. M. W. Pappas, S. Cheng, K. A. Richardson, R. Henning, Y. Kahn, R. Nguyen et al., Phys. Rev. Lett. 127, 081801 (2021).

[14] M. Silva-Feaver et al., IEEE Trans. Appl. Supercond. 27, 1 (2017).

[15] J. W. Brockway, E. D. Carlson, and G. G. Raffelt, Phys. Lett. B 383, 439 (1996); J. A. Grifols, E. Massó, and R. Toldrà, Phys. Rev. Lett. 77, 2372 (1996).

[16] A. Payez et al., J. Cosmol. Astropart. Phys. 02 (2015) 006.

[17] D. Wouters and P. Brun, Phys. Rev. D 86, 043005 (2012); D. Wouters and P. Brun, Astrophys. J. 772, 44 (2013); A. Abramowski et al. (H.E.S.S. Collaboration), Phys. Rev. D 88, 102003 (2013); J. P. Conlon, A. J. Powell, and M. C. D. Marsh, Phys. Rev. D 93, 123526 (2016); M. Ajello et al. (The Fermi-LAT Collaboration), Phys. Rev. Lett. 116, 161101 (2016); M. Berg et al., Astrophys. J. 847, 101 (2017).

[18] M. C. D. Marsh et al., J. Cosmol. Astropart. Phys. 12 (2017) 036.

[19] C. S. Reynolds, M. C. D. Marsh, H. R. Russell, A. C. Fabian, R. Smith, F. Tombesi, and S. Veilleux, Astrophys. J. 890, 59 (2020).
[20] C. Dessert, J. W. Foster, and B. R. Safdi, Phys. Rev. Lett. 125, 261102 (2020).

[21] J. P. Conlon, F. Day, N. Jennings, S. Krippendorf, and F. Muia, Mon. Not. R. Astron. Soc. 473, 4932 (2018).

[22] F. Aharonian et al. (H.E.S.S Collaboration), Astron. Astrophys. 475, L9 (2007); J. Albert et al. (MAGIC Collaboration), Science 320, 1752 (2008); A. De Angelis, M. Roncadelli, and O. Mansutti, Phys. Rev. D 76, 121301 (2007); D. Horns and M. Meyer, J. Cosmol. Astropart. Phys. 02 (2012) 033; M. Meyer, D. Horns, and M. Raue, Phys. Rev. D 87, 035027 (2013).

[23] J. P. Conlon and M. C. D. Marsh, Phys. Rev. Lett. 111, 151301 (2013); S. Angus, J. P. Conlon, M. C. D. Marsh, A. J. Powell, and L. T. Witkowski, J. Cosmol. Astropart. Phys. 09 (2014) 026.

[24] K. Kohri and H. Kodama, Phys. Rev. D 96, 051701 (2017).

[25] T. Moroi, K. Nakayama, and Y. Tang, Phys. Lett. B 783, 301 (2018).

[26] A. Caputo et al., Phys. Rev. D 100, 063515 (2019).

[27] A. V. Gramolin, D. Aybas, D. Johnson, J. Adam, and A. O. Sushkov, Nat. Phys. 17, 79 (2021).

[28] A. G. Dias, A. C. B. Machado, C. C. Nishi, A. Ringwald, and P. Vaudrevange, J. High Energy Phys. 06 (2014) 037; P. W. Graham, I. G. Irastorza, S. K. Lamoreaux, A. Lindner, and K. A. van Bibber, Annu. Rev. Nucl. Part. Sci. 65, 485 (2015); I. G. Irastorza and J. Redondo, Prog. Part. Nucl. Phys. 102, 89 (2018); M. Y. Khlopov, Int. J. Mod. Phys. D 27, 1841013 (2018).

[29] P. Sikivie, Phys. Rev. Lett. 51, 1415 (1983); M. B. Schneider, F. P. Calaprice, A. L. Hallin, D. W. MacArthur, and D. F. Schreiber, Phys. Rev. Lett. 52, 695 (1984); G. Raffelt and L. Stodolsky, Phys. Rev. D 37, 1237 (1988).

[30] L. Tsukada, T. Callister, A. Matas, and P. Meyers, Phys. Rev. D 99, 103015 (2019); C. Palomba, S. D'Antonio, P. Astone, S. Frasca, G. Intini, I. La Rosa, P. Leaci, S. Mastrogiovanni, A. L. Miller, F. Muciaccia et al., Phys. Rev. Lett. 123, 171101 (2019); L. Sun, R. Brito, and M. Isi, Phys. Rev. D 101, 063020 (2020); 102, 089902(E) (2020); K. K. Y. Ng, S. Vitale, O. A. Hannuksela, and T. G. F. Li, Phys. Rev. Lett. 126, 151102 (2021).

[31] S. M. Carroll, G. B. Field, and R. Jackiw, Phys. Rev. D 41, 1231 (1990); S. M. Carroll, Phys. Rev. Lett. 81, 3067 (1998).

[32] A. Andrianov, D. Espriu, F. Mescia, and A. Renau, Phys. Lett. B 684, 101 (2010); D. Espriu and A. Renau, Phys. Rev. D 85, 025010 (2012); T. Fujita, R. Tazaki, and K. Toma, Phys. Rev. Lett. 122, 191101 (2019).

[33] A. C. Melissinos, Phys. Rev. Lett. 102, 202001 (2009).

[34] W. DeRocco and A. Hook, Phys. Rev. D 98, 035021 (2018).

[35] I. Obata, T. Fujita, and Y. Michimura, Phys. Rev. Lett. 121, 161301 (2018).

[36] H. Liu, B. D. Elwood, M. Evans, and J. Thaler, Phys. Rev. D 100, 023548 (2019).

[37] K. Nagano, T. Fujita, Y. Michimura, and I. Obata, Phys. Rev. Lett. 123, 111301 (2019).

[38] D. Martynov and H. Miao, Phys. Rev. D 101, 095034 (2020).

[39] J. Aasi et al., Classical Quantum Gravity 32, 074001 (2015). 
[40] F. Acernese et al., Classical Quantum Gravity 32, 024001 (2015).

[41] K. Somiya, Classical Quantum Gravity 29, 124007 (2012); Y. Aso et al., Phys. Rev. D 88, 043007 (2013); T. Akutsu et al., Prog. Theor. Exp. Phys. (2018), 013F01; T. Akutsu et al., Prog. Theor. Exp. Phys. (2020), ptaa125.

[42] M. Punturo et al., Classical Quantum Gravity 27, 194002 (2010).

[43] B. P. Abbott et al., Classical Quantum Gravity 34, 044001 (2017).

[44] S. Kawamura et al., J. Phys. Conf. Ser. 120, 032004 (2008); S. Kawamura et al., Classical Quantum Gravity 28, 094011 (2011); S. Kawamura et al., Prog. Theor. Exp. Phys. (2021), $05 \mathrm{~A} 105$.

[45] R. Brito, S. Ghosh, E. Barausse, E. Berti, V. Cardoso, I. Dvorkin, A. Klein, and P. Pani, Phys. Rev. Lett. 119, 131101 (2017); N. Kitajima, J. Soda, and Y. Urakawa, J. Cosmol. Astropart. Phys. 10 (2018) 008; C. S. Machado, W. Ratzinger, P. Schwaller, and B. A. Stefanek, J. High Energy Phys. 01 (2019) 053.

[46] B. J. Meers, Phys. Rev. D 38, 2317 (1988); J. Mizuno et al., Phys. Lett. A 175, 273 (1993).
[47] J. W. Foster, N. L. Rodd, and B. R. Safdi, Phys. Rev. D 97, 123006 (2018).

[48] P. F. de Salas and A. Widmark, arXiv:2012.11477.

[49] G. P. Centers, J. W. Blanchard, J. Conrad, N. L. Figueroa, A. Garcon, A. V. Gramolin, D. F. J. Kimball, M. Lawson, B. Pelssers, J. A. Smiga et al., arXiv:1905.13650.

[50] R. W. P. Drever et al., Appl. Phys. B 31, 97 (1983).

[51] A. Yariv and P. Yeh, Photonics: Optical Electronics in Modern Communications (Oxford University Press, New York, 2007).

[52] R. W. P. Drever et al., Gravitational Wave Detectors Using Laser Interferometers and Optical Cavities: Ideas, Principles and Prospects, in Quantum Optics, Experimental Gravity, and Measurement Theory, edited by P. Meystre and M. O. Scully, NATO Advanced Science Institutes Series (Springer US, Boston, MA, 1983), pp. 503-514.

[53] S. Morisaki and T. Suyama, Phys. Rev. D 100, 123512 (2019).

[54] H. J. Kimble, Y. Levin, A. B. Matsko, K. S. Thorne, and S. P. Vyatchanin, Phys. Rev. D 65, 022002 (2001).

[55] D. Budker, P. W. Graham, M. Ledbetter, S. Rajendran, and A. Sushkov, Phys. Rev. X 4, 021030 (2014). 